\title{
Controllable Tunneling of Light through a Quantum-Dot-Molecule Dielectric Film via Electromagnetically Induced Transparency
}

\author{
Ruixi Zeng, Jing Gu, Jianqi Shen* \\ Centre for Optical and Electromagnetic Research, State Key Laboratory for Modern Optical Instrumentation, Zijingang Campus, \\ Zhejiang University, Hangzhou, China \\ Email: *jqshen@zju.edu.cn
}

How to cite this paper: Zeng, R.X., Gu, J. and Shen, J.Q. (2017) Controllable Tunneling of Light through a Quantum-Dot-Molecule Dielectric Film via Electromagnetically Induced Transparency. Optics and Photonics Journal, 7, 49-67. https://doi.org/10.4236/opj.2017.78B008

Received: May 19, 2017

Accepted: August 7, 2017

Published: August 10, 2017

\begin{abstract}
Since discrete multilevel transitions of quantum-dot molecules driven by external electromagnetic fields can exhibit quantum coherence effects, such an optical characteristic can be utilized to control propagation of electromagnetic wave through a quantum-dot molecule dielectric film. Since inner-dot tunneling in quantum-dot molecules can be controlled by a gate voltage, destructive quantum coherence among multilevel transitions in quantum-dot molecule would give rise to EIT (electromagnetically induced transparency). In this report, we shall investigate controllable on- and off-resonance tunneling effects of an incident electromagnetic wave through such a quantum-dot-molecule dielectric film, of which the optical response is tuned by the switchable gate voltage. We have found from the theoretical mechanism that a high gate voltage can cause the EIT phenomenon of quantum-dot-molecule systems, and under the condition of on-resonance light tunneling through the thin film, the probe field will propagation without loss if the probe frequency detuning is zero. By taking advantage of these effects sensitive to the tunable gate voltage, such quantum coherence would be integrated in certain photonic structures, and some devices such as photonic switching and transistors can be designed. Transient evolution of optical characteristics in the quantum-dot-molecule dielectric film (once the tunable gate voltage is turned on or off) is also considered in this report.
\end{abstract}

\section{Keywords}

Resonant Tunneling, Quantum-Dot Molecules, Quantum Coherence, Controllable Transmission 


\section{Introduction}

Light propagation control is one of the key technologies with the development of photonic and optical science. Over the past two decades, utilizing one light to control another light has attracted a lot of interest of a group of researchers [1] [2] [3] [4] [5]. In quantum optics, quantum coherence can be used to realize such applications. For example, quantum coherence in atomic physics has captured intensive attention because it exhibits some novel phenomena in various multi-level atomic systems [3]-[9]. All of these effects can be found in three or four level atomic systems, which control the weak probe field through constructive or destructive quantum coherence among transitions driven by one or two applied control lights. Quantum coherence effects are often observed in neutral alkali-metal atomic vapors [3]-[8]. However, there are also some discrete energy level structures in solid materials, which may also be used to realize quantum coherence. One of them is the material of semiconductor quantum dots [10] [11] [12]. As is well known, in a quantum dot, electrons and holes should only be in some specific states of quantum mechanical wave functions, and this would cause the effect of multilevel transitions. That is to say, lossless propagation of a probe field in such a quantum-dot quantum coherent medium can be achieved (electromagnetically induced transparency, EIT) if we tune the frequency of the probe field resonant with the quantum-dot energy level transition. In this case EIT effect results from the destructive quantum coherence among the quantumdot three-level transitions. Besides, we should also take into account the quantum-dot molecules [13] [14] [15] [16]. A quantum-dot molecule is a system of two or more coupled quantum dots, where coherent electronic states form through inter-dot tunneling [17] [18]. There have been many interesting applications and effects realized in quantum-dot molecular media, e.g., optical bistability [19] [20], slow light [21] [22], resonance fluorescence [23], electron-hole excitons [24], quantum interference and unusual optical responses [25], as well as tripartite hybrid quantum entanglement [26].

In this work, we will discuss the propagation effect controlled by a tunable gate voltage through a quantum-dot molecule dielectric film. Our study is performed based on the work of Zohravi and M. Mahmoudi [18]. We shall focus on the controllable tunneling effect via quantum coherence, including the multilevel transitions of quantum-dot molecules. The transitions of quantum-dot molecules are both driven by a gate voltage (playing a role of controlling light in the conventional EIT) and by an incident electromagnetic wave (playing a role of probe light in the conventional EIT), and then the transmission characteristics of the incident electromagnetic wave can be controlled by the gate voltage. We expect that this tunable and sensitive optical response driven by quantum coherence in multi-level quantum-dot molecule systems may open prospects in new domains for designing devices, e.g., microcircuits in integrated photonics/optics.

The central working mechanism of this work has been published in the litera- 
ture [27]. However, this paper [27] may be quite overelaborate for understanding because there are various tedious physical details. In the present conference report, we shall review briefly the physical mechanism and the main results of this work on "quantum-coherence assisted tunable on-and off-resonance tunneling through a quantum-dot-molecule dielectric film".

\section{Theoretical Mechanism of Controllable Tunneling of Light through a Quantum-Dot-Molecule Dielectric Film}

Novel optical and electromagnetic responses relevant to quantum coherence can be exhibited through level transitions caused by phase coherence in quantumdot systems, including quantum-dot molecular systems. A three-level system model can be used to describe the interaction between an incident probe light and a quantum-dot molecule, in which the light-molecule coupling is manipulated through a tunable gate voltage, e.g., an $\Lambda$-configuration three-level system (see Figure 1(a)) consists of two lower level $|0\rangle,|2\rangle$ and one upper level $|1\rangle$. As is well known, in a traditional atomic EIT, a control light couples the pair of $|2\rangle-|1\rangle$ and a weak probe light drives the transition of $|0\rangle-|1\rangle$. Now in the present quantum-dot molecule (see Figure 1(a)), a low-frequency gate voltage plays the role of the control light, which excites the $|2\rangle-|1\rangle$ transition (causing inner-dot electron tunneling between two quantum dots in a quantum-dot molecular system [18]).

We define the frequency detuning of control light and probe light as $\Delta_{c}=\omega_{12}-\omega_{c}$ and $\Delta_{p}=\omega_{10}-\omega_{p}$, respectively, where $\omega_{12}$ and $\omega_{10}$ represent the level transition frequencies, and $\omega_{c}, \omega_{p}$ denote the angular frequencies of

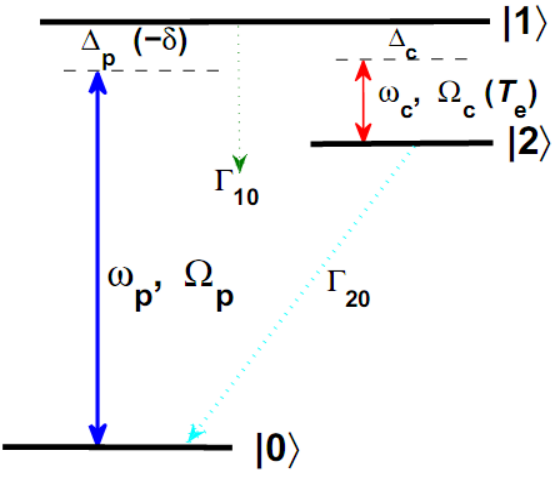

(a)

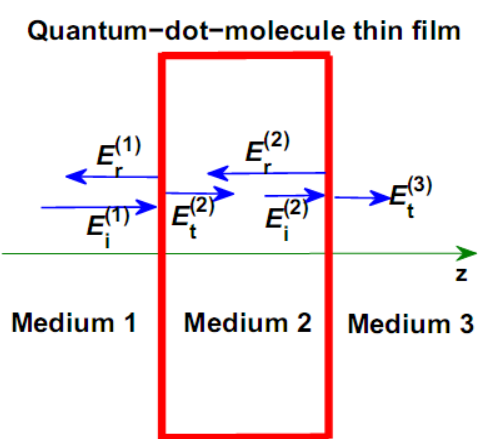

(b)

Figure 1. Schematic diagrams of three-level configuration in a quantum-dot molecule (a) and the propagation of light in quantum-dot molecule film (b). The three-level $\Lambda$-configuration quantum-dot molecule system consists of two quantum dots (a), i.e., $|0\rangle-|1\rangle$ and $|2\rangle-|1\rangle$ systems. An incident probe light $\left(\Omega_{p}\right)$ drives the $|0\rangle-|1\rangle$ transition and a low-frequency gate voltage $\left(T_{e}\right)$ couples the $|2\rangle-|1\rangle$ level pair. A quantum-dot molecule film or dielectric slab (b) can be used to control the incident weak probe light propagation via both EIT (electromagnetically induced transparency) and on/off-resonance light tunneling effect though the quantum-dot molecule film (medium 2). 
the control and probe fields, respectively. Since the incident probe light $\left(\Omega_{p}\right)$ drives the $|0\rangle-|1\rangle$ transition and the low-frequency gate voltage $\left(T_{e}\right)$ couples the $|2\rangle-|1\rangle$ level pair, by using the Hamiltonian of such interactions and according to the quantum mechanical Schrödinger equation, we can derive the equation of motion of the density matrix elements $\rho_{i j}$ of the present three-level quantum-dot molecule system [27]:

$$
\begin{aligned}
& \mathrm{d} \dot{\rho}_{00} / \mathrm{d} t=\gamma_{10} \rho_{11}+\gamma_{20} \rho_{22}-\frac{i}{2}\left(\Omega_{p} \rho_{01}-\Omega_{p}^{*} \rho_{10}\right), \\
& \mathrm{d} \dot{\rho}_{22} / \mathrm{d} t=\gamma_{12} \rho_{11}-\gamma_{20} \rho_{22}-\frac{i}{2}\left(\Omega_{c} \rho_{21}-\Omega_{c}^{*} \rho_{12}\right), \\
& \mathrm{d} \dot{\rho}_{11} / \mathrm{d} t=-\left(\gamma_{10}+\gamma_{12}\right) \rho_{11}+\frac{i}{2}\left(\Omega_{p} \rho_{01}+\Omega_{c} \rho_{21}\right)-\frac{i}{2}\left(\Omega_{p}^{*} \rho_{10}+\Omega_{c}^{*} \rho_{12}\right), \\
& \mathrm{d} \dot{\rho}_{01} / \mathrm{d} t=-\left(\Gamma_{01}-i \Delta_{p}\right) \rho_{01}-\frac{i}{2}\left(\Omega_{p}^{*} \rho_{00}+\Omega_{c}^{*} \rho_{02}\right)+\frac{i}{2} \Omega_{p}^{*} \rho_{11}, \\
& \mathrm{~d} \dot{\rho}_{21} / \mathrm{d} t=-\left(\Gamma_{21}-i \Delta_{c}\right) \rho_{21}-\frac{i}{2}\left(\Omega_{p}^{*} \rho_{20}+\Omega_{c}^{*} \rho_{22}\right)+\frac{i}{2} \Omega_{c}^{*} \rho_{11}, \\
& \mathrm{~d} \dot{\rho}_{02} / \mathrm{d} t=-\left[\Gamma_{02}-i\left(\Delta_{p}-\Delta_{c}\right)\right] \rho_{02}-\frac{i}{2} \Omega_{c} \rho_{01}+\frac{i}{2} \Omega_{p}^{*} \rho_{12} .
\end{aligned}
$$

The Rabi frequency of the weak probe light and the gate voltage are defined as $\Omega_{p}=\wp_{10} \varepsilon_{p} / \hbar$ and $\Omega_{c}=2 T_{e} / \hbar$, respectively, where $\varepsilon_{p}$ denotes the envelop of the probe light and $T_{e}$ is the gate-voltage electric potential energy of the $|1\rangle$ - $|2\rangle$ transition dipole in the quantum-dot molecule. From the density matrix Equation (1), we can see when a destructive quantum coherence condition

$$
\Omega_{p} \rho_{01}+\Omega_{c} \rho_{21}=0
$$

for $|1\rangle-|2\rangle$ transition and $|0\rangle-|1\rangle$ transition is fulfilled, the coherent population trapping (CPT) will be achieved (e.g., $\rho_{11} \rightarrow 0$ ). That is to say, the whole driving contribution, i.e., the sum of $\Omega_{p} \rho_{01}$ (caused by the incident electromagnetic wave) and $\Omega_{p} \rho_{01}$ (caused by the gate voltage), vanishes through the interference pathways $(|2\rangle-|1\rangle$ and $|0\rangle-|1\rangle)$, and so the upper level $|2\rangle$ seems to be absent. There is no net interaction (i.e., no net $|0\rangle-|1\rangle$ transition) driven by the incident electromagnetic wave (probe light), and the quantum-dot molecule dielectric is transparent to this incident electromagnetic wave. Such an effect can be called "quantum-dot molecular EIT".

Now we can obtain the steady solution of the equation, noting that in the equation of $\mathrm{d} \rho_{01} / \mathrm{d} t$ the small term $\frac{i}{2} \Omega_{p}^{*} \rho_{11}$ can be ignored. The result of the steady solution that characterizes the $|0\rangle-|1\rangle$ transition is given by

$$
\rho_{10}=\frac{\frac{1}{2}\left[-\left(\Delta_{p}-\Delta_{c}\right)+i \Gamma_{20}\right] \Omega_{p} \rho_{00}}{\left(\Gamma_{10}+i \Delta_{p}\right)\left[\Gamma_{20}+i\left(\Delta_{p}-\Delta_{c}\right)\right]+\frac{1}{4} \Omega_{c}^{*} \Omega_{c}} .
$$

In order to comply with the "notations and conventions" of Zohravi and M. Mahmoudi [18], we will choose the probe frequency detuning $\Delta_{\mathrm{p}} \rightarrow-\delta$ and 
$\frac{\hbar^{2}}{4} \Omega_{c}^{*} \Omega_{c} \rightarrow T_{e}^{2}$. Since the Rabi frequency of the probe field is $\Omega_{\mathrm{p}} \propto \varepsilon_{p}$ (the electric field strength of the incident electromagnetic wave), the electric susceptibility $\chi$ of a dielectric film doped with the present quantum-dot molecules is proportional to the density matrix element $\rho_{10}$ :

$$
\chi=A \hbar \frac{\hbar \delta+i \hbar \Gamma_{20}}{\left(\hbar \Gamma_{10}-i \hbar \delta\right)\left(\hbar \Gamma_{20}-i \hbar \delta\right)+T_{e}^{2}} .
$$

If the gate voltage $T_{e}$ is large enough (e.g., $T_{e}^{2} \gg(\hbar \delta)^{2}$ or $\left.T_{e}^{2} \gg\left|\left(\hbar \Gamma_{10}-i \hbar \delta\right)\left(\hbar \Gamma_{20}-i \hbar \delta\right)\right|\right)$ and the probe frequency detuning $\delta=0$, the electric susceptibility $\chi$ will approach $\frac{A \hbar\left(\hbar \delta+i \hbar \Gamma_{20}\right)}{T_{e}^{2}} \rightarrow 0$ (the dephasing rate $\Gamma_{20}$ is negligibly small). This means that a resonant laser beam will not be absorbed by the present quantum coherent system (due to EIT effect).

Next we shall discuss the reflection and transmission of the incident probe light in the thin film system. Figure 1(b) and Figure 2 shows that a uniform electromagnetic plane wave (probe field) which travels along z-direction in medium $1\left(\varepsilon_{1}, \mu_{1}\right)$ is normally incident on the left boundary of medium $2\left(\varepsilon_{2}, \mu_{2}\right)$, then it passes through medium 2 and finally it is transmitted into medium 3 $\left(\varepsilon_{3}, \mu_{3}\right)$. The reflection and transmission occurs on both interfaces of medium 2 (quantum-dot molecular dielectric film). So by using the theory of film optics and the electromagnetic boundary conditions [28], the reflection coefficient at the left interface of medium 2 is

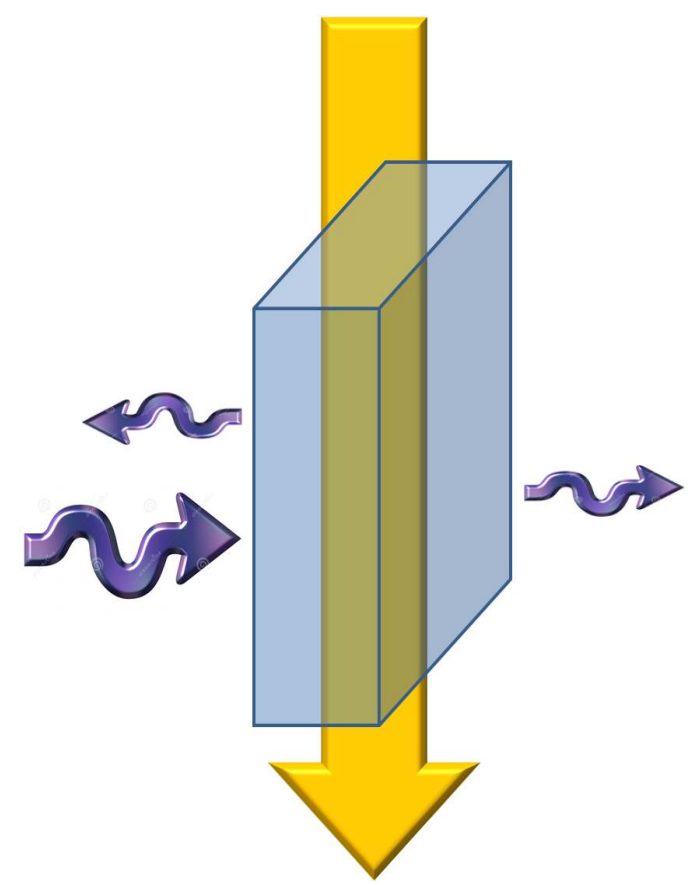

Figure 2. Schematic diagram of the propagation of a probe light through a quantum-dot molecule film. The probe field is incident on the film, and is reflected back and transmitted through the film. The thick arrow represents the control field or the voltage gate. 


$$
r=\frac{-\frac{i}{2}\left(\frac{1}{\sqrt{\varepsilon_{2}}}-\sqrt{\varepsilon_{2}}\right) \sin \beta d}{\cos \beta d-\frac{i}{2}\left(\frac{1}{\sqrt{\varepsilon_{2}}}+\sqrt{\varepsilon_{2}}\right) \sin \beta d}
$$

and the transmission coefficient of the film is

$$
t=\frac{E_{t}^{(3)}}{E_{i}^{(1)}}=\frac{\exp (i \beta d)}{\cos \beta d-\frac{i}{2}\left(\frac{1}{\sqrt{\varepsilon_{2}}}+\sqrt{\varepsilon_{2}}\right) \sin \beta d} .
$$

In Equations (5) and (6), $d$ denotes the quantum-dot molecular film thickness and $\beta$ is the phase constant $\left(\beta=\sqrt{\varepsilon_{2}} \omega / c\right)$ in medium 2 (quantum-dot molecular film). In the following illustrative example, medium 1 and medium 3 are vacuum.

Now the theoretical model for "quantum-coherence assisted tunable on- and off-resonance tunneling through a quantum-dot-molecule dielectric film" has been established, and in the following sections, we shall give some numerical examples of controllable tunneling of light through the quantum-dot-molecule dielectric film.

\section{Numerical Example of Quantum Coherence for Manipulating Electromagnetic Wave Transmission}

The tunable light tunneling through a quantum-coherent dielectric film doped with quantum-dot molecules will be considered in what follows. The definitions of "on resonance" and "off resonance" are given as follows: when the thin film thickness $d$ agrees with $d=2 m \lambda_{0} /\left(4 \sqrt{\varepsilon_{b}}\right)$, where $\lambda_{0}$ is the wavelength of the incident probe wave in vacuum, it can be referred to as "on-resonance" light tunneling, because the thin film thickness $d$ is an integral multiple of half wavelength in the bulk dielectric 2 (thin film). However, when the thin film thickness $d$ is $d=(2 m+1) \lambda_{0} /\left(4 \sqrt{\varepsilon_{b}}\right)$, this can be called the condition of "off-resonance" light tunneling. Here, $m$ is an integer. In the following numerical example, the parameters of the quantum-dot molecules in the electric susceptibility $\chi$ are given by $\Gamma_{10}=5.5 \mathrm{meV} / \hbar, \Gamma_{20}=5.0 \mu \mathrm{eV} / \hbar, A=5.0 \mathrm{meV} / \hbar$. We assume the gate-voltage frequency detuning $\Delta_{c}=0$. These parameters will be used throughout the present paper. The dispersion behavior of the tunable electric susceptibility $\chi$ of the quantum-dot-molecular dielectric is plotted in Figure 3 and Figure 4. The susceptibility $\chi$ of the quantum-dot-molecule dielectric can be controlled by tuning the gate voltage $T_{e}$, namely, the gate voltage can be turned on or off, and $T_{e}$ can be chosen as any magnitudes. The optical characteristics of the quantum-dot-molecular dielectric film can be adjustable with the tunable gate voltage $T_{e}$.

The dispersion of $r$ (reflection coefficient), $R$ (reflectance) and $t$ (transmission coefficient), $T$ (transmittance) of the thin film in the cases of both on- 

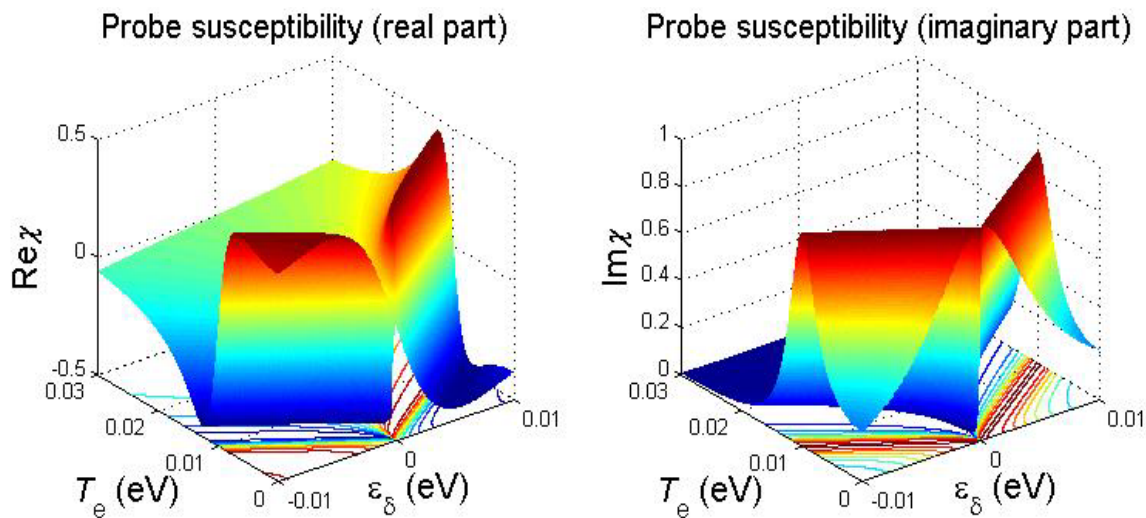

Figure 3. Both the real and imaginary parts of the susceptibility $\chi$ of the quantumdot-molecule dielectric depending on both the gate voltage $T_{e}$ and the probe frequency detuning $\delta\left(\hbar \delta=\hbar \omega_{p}-\hbar \omega_{10}=\varepsilon_{\delta}\right)$. The single quantum-dot gate voltage $T_{e}$ is defined as the electric potential energy of $|2\rangle-|1\rangle$ transition electric dipole in an individual quantum-dot molecule.
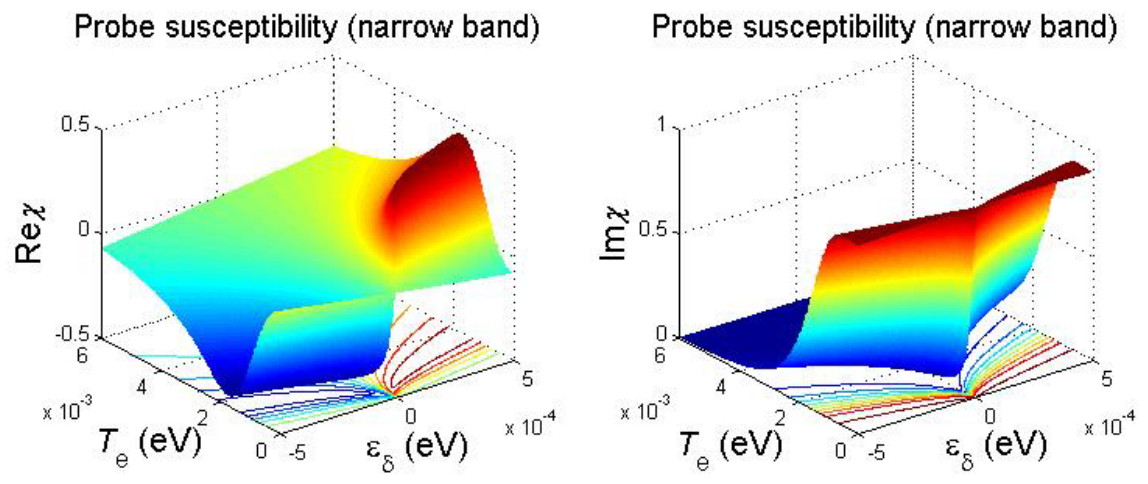

Probe susceptibility (broader band)
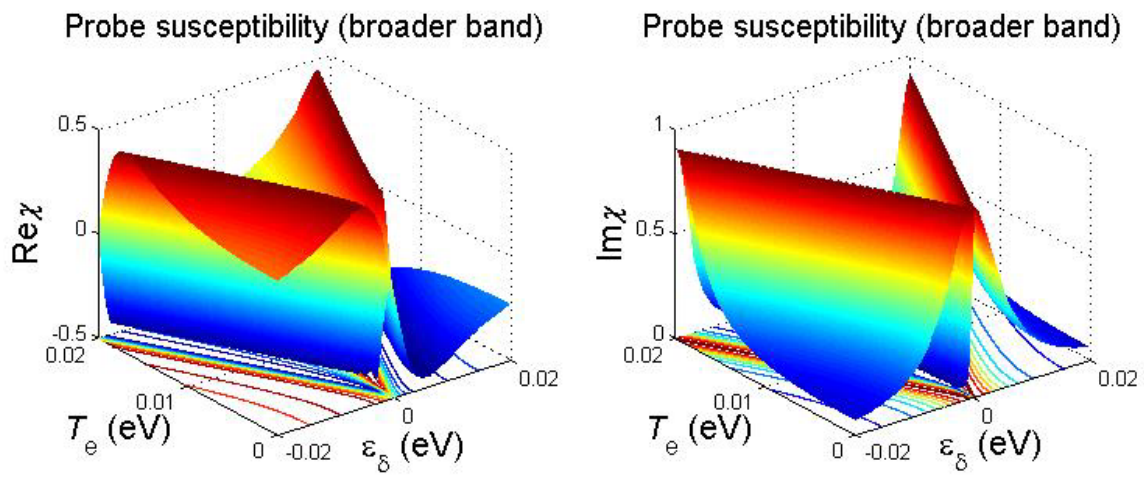

Figure 4. Both the real and imaginary parts of the susceptibility $\chi$ of the quantum-dotmolecule dielectric depending on both the gate voltage $T_{e}$ and the probe frequency detuning $\delta\left(\hbar \delta=\hbar \omega_{p}-\hbar \omega_{10}=\varepsilon_{\delta}\right)$.

and off-resonance tunnelling are plotted in Figure 5 and Figure 6, where we have considered the effect of quantum coherence in the cases of small gate voltage $T_{e}=0.0$ and $2.0 \mathrm{meV}$. EIT does not occur since the gate voltage $T_{e}$ 


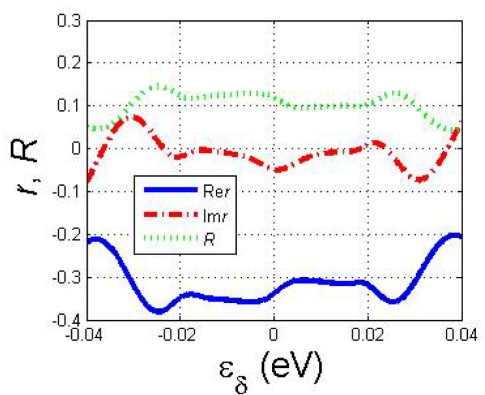

(a)

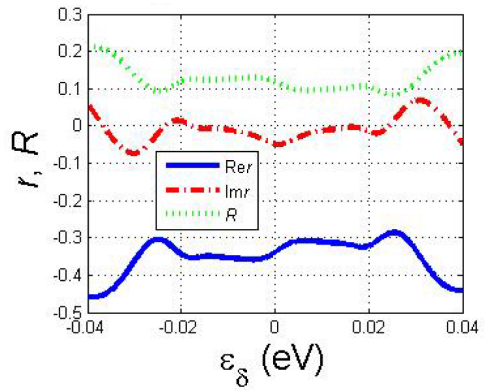

(c)

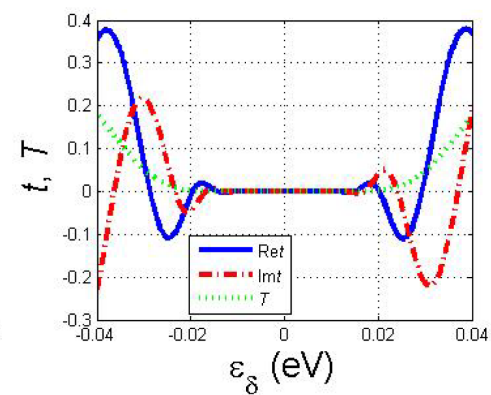

(b)

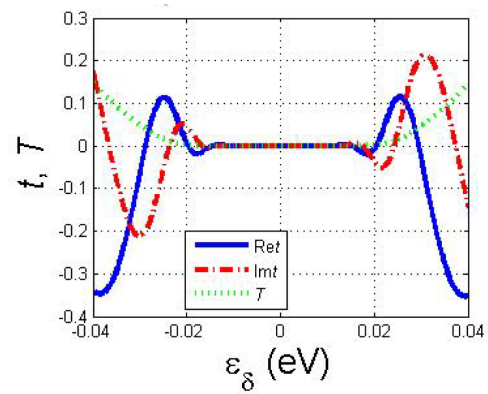

(d)

Figure 5. The dispersion characteristics of the reflection coefficient $r$, reflectance $R$ and transmission coefficient $t$, transmittance $T$ of the quantum-dot-molecule dielectric film when the gate voltage is turned off $\left(T_{e}=0\right)$. In the film thickness $d=2 m \lambda_{0} /\left(4 \sqrt{\varepsilon_{b}}\right)$ (on resonance) and $d=(2 m+1) \lambda_{0} /\left(4 \sqrt{\varepsilon_{b}}\right)$ (off resonance), the integer $m=60$. Since the gate voltage is zero, there is no EIT effect at the probe resonance frequency $(\delta=0)$. The two-level resonant absorption (caused by $|0\rangle-|1\rangle$ transition) reduces both the reflectance and transmittance, e.g., even when the transmittance $T$ vanishes, the reflectance $\Omega_{p} \rho_{01}+\Omega_{c} \rho_{21}=0$ is small because of $|0\rangle-|1\rangle$ resonant absorption. (a) $T_{e}=$ $0.0 \mathrm{meV}$, on resonance; (b) $T_{e}=0.0 \mathrm{meV}$, on resonance; (c) $T_{e}=0.0 \mathrm{meV}$, off resonance; (d) $T_{e}=0.0 \mathrm{meV}$, off resonance.

is zero or small. For the following figures, we have chosen the background permittivity of the dielectric film $\varepsilon_{b}=4$, the vacuum wavelength $\lambda_{0}=3 \times 10^{8} /\left(242 \times 10^{9}\right) \mathrm{m}$ for the incident probe light, and the integer $m=60$ in the film thickness $d=2 m \lambda_{0} /\left(4 \sqrt{\varepsilon_{b}}\right)$ (on resonance) and $d=(2 m+1) \lambda_{0} /\left(4 \sqrt{\varepsilon_{b}}\right) \quad$ (off resonance). The parameters of $\varepsilon_{b}=4$ and $\lambda_{0}=3 \times 10^{8} /\left(242 \times 10^{9}\right) \mathrm{m}$ will be used throughout this work. Since the gate voltage is zero in Figure 5, there exhibits no EIT effect at the probe resonance frequency $(\delta=0)$. In this case, both the reflectance $R$ and the transmittance $T$ are reduced by the two-level resonant absorption (caused by the $|0\rangle-|1\rangle$ transition).

In the above figures, the EIT effect is not dominant because the gate voltage $T_{e}$ is low. In what follows, we shall show that the gate-voltage-driven inter-dot tunneling in a quantum-dot molecule can enable the typical quantum coherence effects (i.e., EIT) to be exhibited. The behavior of tunable on- and off-resonance light tunneling through a quantum coherent quantum-dot-molecule dielectric film, where EIT is involved, will be addressed below. 


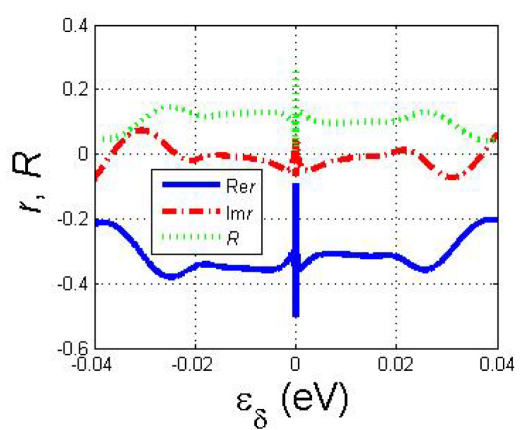

(a)

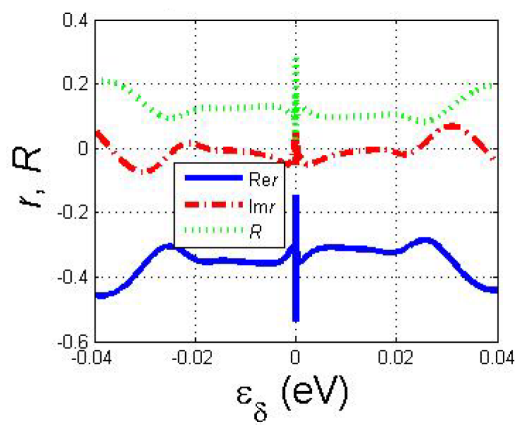

(c)

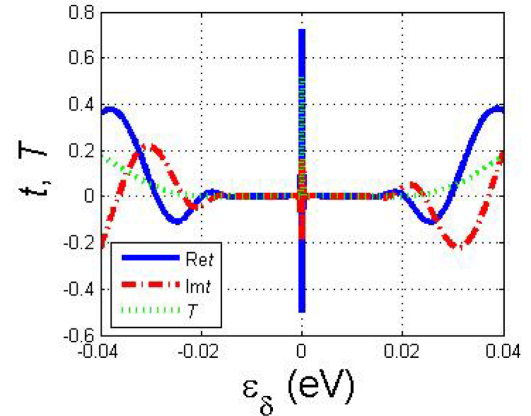

(b)

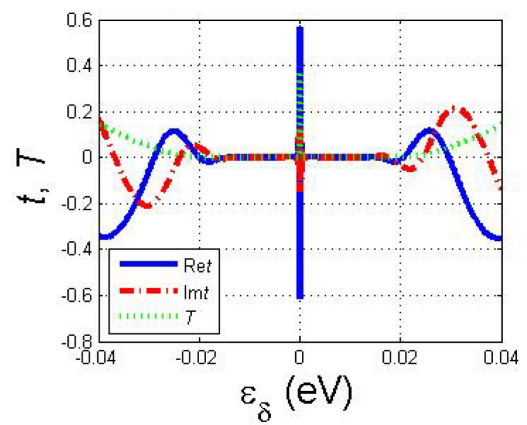

(d)

Figure 6. The reflection and transmission spectrum of the thin film in the cases of both on- and off-resonance tunneling, i.e., the effect caused by two-level absorption $(|0\rangle-|1\rangle$ transition) at small detuning frequency $\delta$ and small gate voltage $T_{e}$. Since EIT is not exhibited in the present quantum-dot-molecule systems at the probe detuning frequency $\delta=0$ when the gate voltage $T_{e}$ is small, the transmittance $T$ is negligibly small. The reflectance $R$ of the probe field at the left interface of the thin film is also small because of strong absorption due to two-level resonance $(|0\rangle-|1\rangle$ transition). In the film thickness $d=2 m \lambda_{0} /\left(4 \sqrt{\varepsilon_{b}}\right)$ (on resonance) and $d=(2 m+1) \lambda_{0} /\left(4 \sqrt{\varepsilon_{b}}\right)$ (off resonance), the integer $m=60$. (a) $T_{e}=2.0 \mathrm{meV}$, on resonance; (b) $T_{e}=2.0 \mathrm{meV}$, on resonance; (c) $T_{e}=2.0 \mathrm{meV}$, off resonance; (d) $T_{e}=2.0 \mathrm{meV}$, off resonance.

The reflection and transmission spectra of the thin film in the case of on-resonance tunneling with the film thickness $d=2 m \lambda_{0} /\left(4 \sqrt{\varepsilon_{b}}\right)$ are plotted in Figure 7 and Figure 8, respectively. It can be found that when the gate voltage $T_{e}$ increases, the EIT effect occurs at the probe resonance frequency ( $\left.\varepsilon_{\delta}=\hbar \delta=\hbar \omega_{p}-\hbar \omega_{10}=0\right)$. This gives rise to the resonant tunneling of the probe light, and the reflectance $R$ vanishes at the probe detuning frequency $\delta=0$.

The reflection and the transmission spectra of the thin film in the case of off-resonance tunneling with the film thickness $d=(2 m+1) \lambda_{0} /\left(4 \sqrt{\varepsilon_{b}}\right)$ are illustrated in Figure 9 and Figure 10, respectively. In this off-resonance tunneling of the probe light, the transmittance $T$ of the probe light is relatively low. Though there is EIT effect to the probe light, the film thickness $d=(2 m+1) \lambda_{0} /\left(4 \sqrt{\varepsilon_{b}}\right)$, which agrees with the condition of off-resonance tunneling, leads to a small transmittance. 


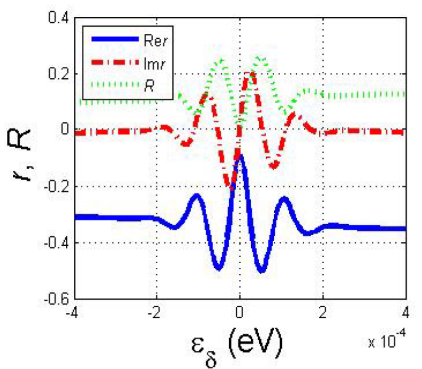

(a)

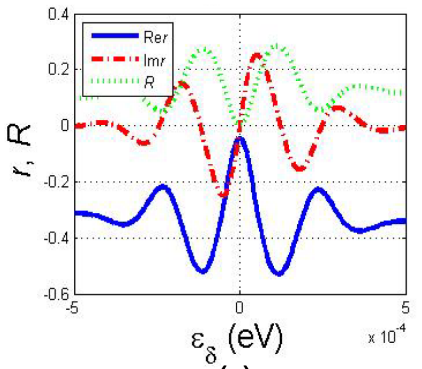

(c)

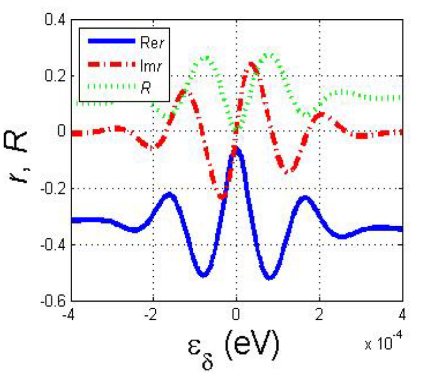

(b)

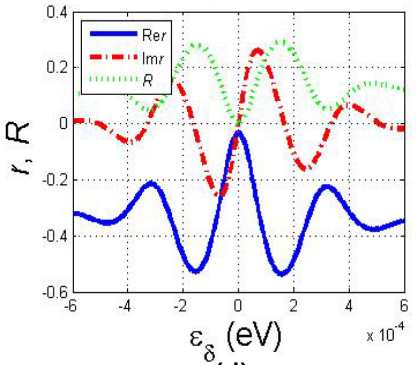

(d)

Figure 7. The reflection spectrum of the thin film in the case of on-resonance light tunneling with the film thickness $d=2 m \lambda_{0} /\left(4 \sqrt{\varepsilon_{b}}\right)$. Here the integer $m=60$. Since EIT effect occurs at the probe resonance frequency $\left(\varepsilon_{\delta}=\hbar \delta=\hbar \omega_{p}-\hbar \omega_{10}=0\right)$, this leads to the resonant tunneling of the probe light, and the reflectance $R$ vanishes at the probe detuning frequency $\delta=0$. (a) $T_{e}=2.0 \mathrm{meV}$; (b) $T_{e}=2.5 \mathrm{meV}$; (c) $T_{e}=3.0 \mathrm{meV}$; (d) $T_{e}=$ $3.5 \mathrm{meV}$.

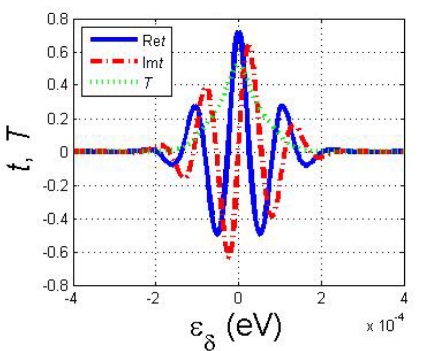

(a)

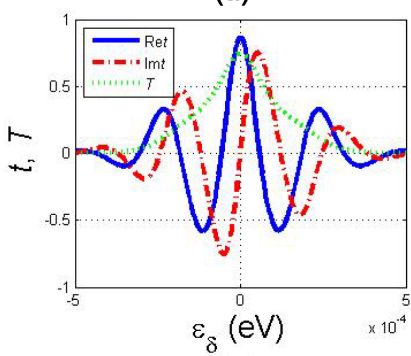

(c)

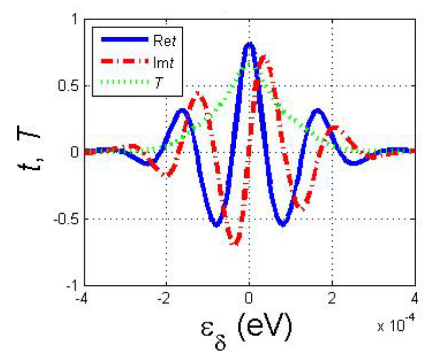

(b)

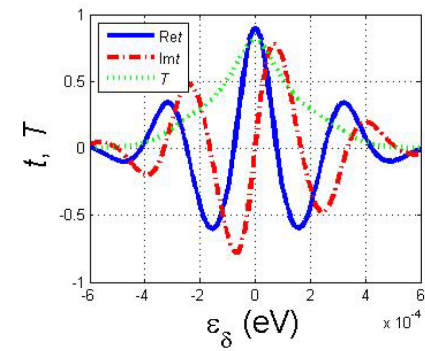

(d)

Figure 8. The transmission spectrum of the thin film in the case of on-resonance tunneling with the film thickness $d=2 m \lambda_{0} /\left(4 \sqrt{\varepsilon_{b}}\right)$. Here, the integer $m=60$. Since EIT effect occurs at the probe resonance frequency $\left(\varepsilon_{\delta}=\hbar \delta=\hbar \omega_{p}-\hbar \omega_{10}=0\right)$, this gives rise to the resonant tunneling of the probe light. The transmittance $T$ through the film becomes large when the gate voltage $T_{e}$ increases (at the probe detuning frequency $\delta=0$ ). (a) $T_{e}=2.0 \mathrm{meV}$; (b) $T_{e}=2.5 \mathrm{meV}$; (c) $T_{e}=3.0 \mathrm{meV}$; (d) $T_{e}=3.5 \mathrm{meV}$. 


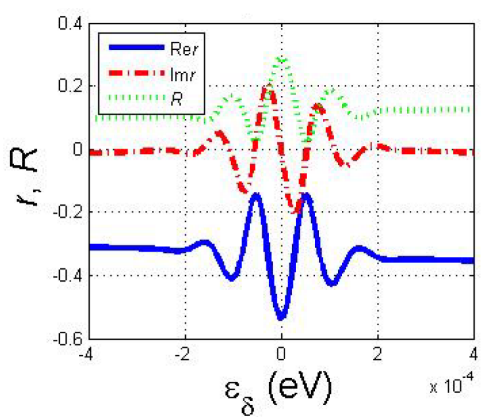

(a)

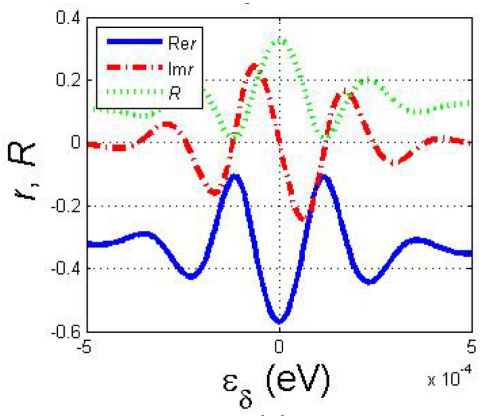

(c)

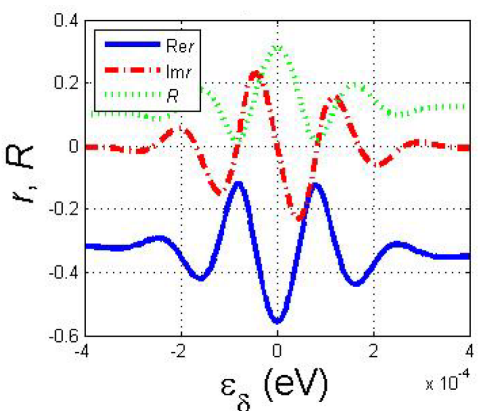

(b)

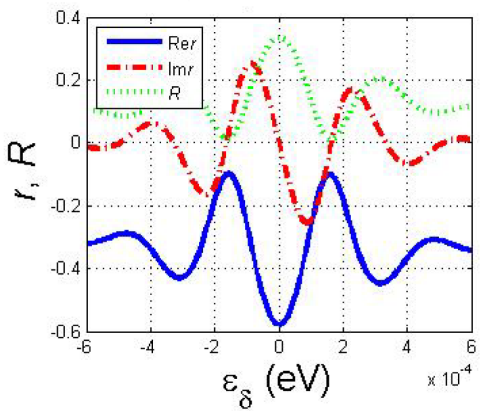

(d)

Figure 9. The reflection spectrum of the thin film in the case of off-resonance tunneling with the film thickness $d=(2 m+1) \lambda_{0} /\left(4 \sqrt{\varepsilon_{b}}\right)$. Here, the integer $m=60$. In this case, the EIT occurs. However, the film thickness satisfies the off-resonance condition. (a) $T_{e}=$ $2.0 \mathrm{meV}$; (b) $T_{e}=2.5 \mathrm{meV}$; (c) $T_{e}=3.0 \mathrm{meV}$; (d) $T_{e}=3.5 \mathrm{meV}$.

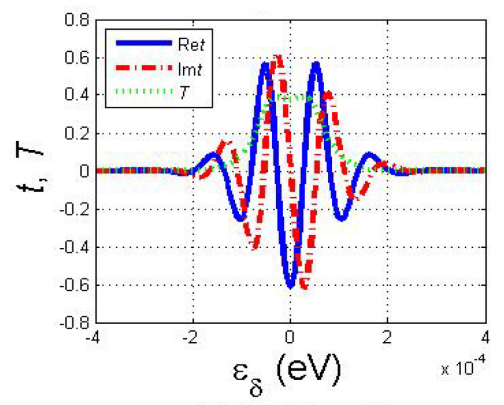

(a)

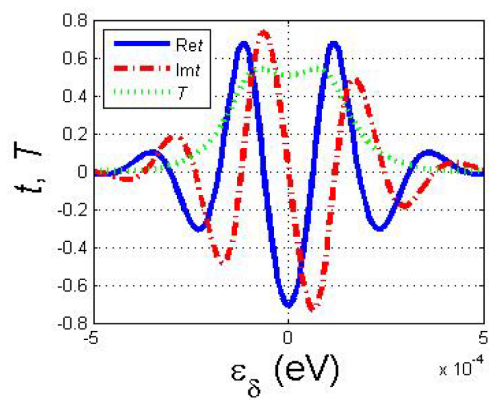

(c)

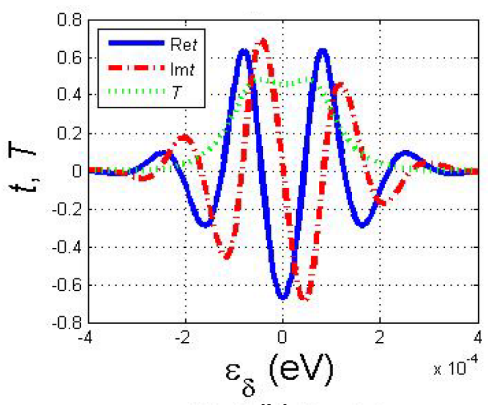

(b)

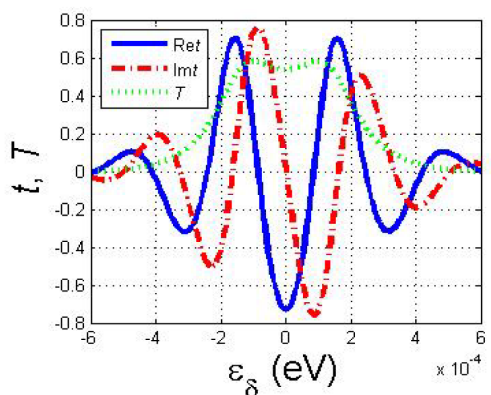

(d)

Figure 10. The transmission spectrum of the thin film in the case of off-resonance tunneling with the film thickness $d=(2 m+1) \lambda_{0} /\left(4 \sqrt{\varepsilon_{b}}\right)$. Here, the integer $m=60$. Since this is the off-resonance tunneling of the probe light, the transmittance $T$ of the probe light is low. (a) $T_{e}=2.0 \mathrm{meV}$; (b) $T_{e}=2.5 \mathrm{meV}$; (c) $T_{e}=3.0 \mathrm{meV}$; (d) $T_{e}=3.5 \mathrm{meV}$. 
Since there are some tunable parameters, e.g., the gate-voltage Rabi frequency and gate-voltage angular frequency, the optical response of quantum-dot molecular systems can be modified by external control fields (e.g., gate voltage), and the tunable reflection and transmission characteristics of the quantum-dot molecular film in the states of on- and off-resonance light tunneling can, therefore, be utilized to design some devices such as photonic switching and transistors.

It can be found in Figure 10 that when the probe frequency detuning ( $\varepsilon_{\delta}=\hbar \delta=\hbar \omega_{p}-\hbar \omega_{10}$ ) becomes large, the transmittance $T$ tends to zero because the EIT phenomenon in the quantum-dot molecular dieclectric is absent. The larger gate voltage $T_{e}$ will give rise to relatively large transmittance $T$. For example, the transmittance $T$ in the case of $T_{e}=3.0 \mathrm{meV}$ is larger than that in the case of $T_{e}=2.0 \mathrm{meV}$ at each probe frequency detuning. It should also be noted that the EIT dispersion is quite strong compared with most of the electromagnetic dielectrics. In general, its disepesion $(\mathrm{d} \chi / \mathrm{d} \omega)$ is five orders of magnitude larger than that of metals. This, therefore, implies that the optical characteristics of the present quantum-dot molecular dieclectric is extremely senstitive to the incident probe light frequency in the narrow frequency band close to the resonance frequency $\left(\varepsilon_{\delta}=\hbar \delta=\hbar \omega_{p}-\hbar \omega_{10}=0\right)$.

\section{The Tunable Dispersion Characteristics of the Reflection and Transmission Spectra in Quantum Coherence for Manipulating Electromagnetic Wave Transmission}

The three-dimeniosional dispersion characteristics of the reflection and transmission spectra of the thin film, in which the film thickness (defined as $\left.d=2 m \lambda_{0} /\left(4 \sqrt{\varepsilon_{b}}\right)\right)$ and the probe frequency detuning (defined as $\left.\varepsilon_{\delta}=\hbar \delta=\hbar \omega_{p}-\hbar \omega_{10}\right)$ are the two variables, have been plotted in Figures 11-13. In Figure 11, the EIT effect can occur when the probe frequency detuning $\varepsilon_{\delta}=\hbar \delta=\hbar \omega_{p}-\hbar \omega_{10}=0$ and the gate voltage $T_{e}=3.0 \mathrm{meV}$, and the transmittance $T$ can increase and the reflectance $R$ decreases. In Figure 12, the gate voltage is relatively small $\left(T_{e}=1.0 \mathrm{meV}\right)$, and the resonant tunneling effect of the probe light cannot be exhibited. Whereas, in Figure 13, the gate voltage becomes large $\left(T_{e}=3.0 \mathrm{meV}\right)$, the resonant tunneling effect of the probe light can be exhibited at the probe frequency detuning $\varepsilon_{\delta}=\hbar \delta=\hbar \omega_{p}-\hbar \omega_{10}=0$.

The three-dimeniosional dispersion characteristics of the reflection and transmission spectrum of the thin film, in which the film thickness is defined as $d=2 m \lambda_{0} /\left(4 \sqrt{\varepsilon_{b}}\right) \quad$ (on resonance) and $d=(2 m+1) \lambda_{0} /\left(4 \sqrt{\varepsilon_{b}}\right)$ (off resonance) with $m=40$, have been plotted in Figure 14 and Figure 15. The on- and offresonance conditions for the film thickness exhibit different tunable dispersion characteristics. For example, when the gate voltage $T_{e}$ is large at the probe frequency detuning $\varepsilon_{\delta}=\hbar \delta=\hbar \omega_{p}-\hbar \omega_{10}=0$, the EIT of the probe field can be exhibited. Under this condition, in the case of on resonance, the resonant tunneling of the probe field will occur, namely, the reflectance $R$ is small and the transmittance $T$ approaches unity. In contrast, in the case of off resonance, the 

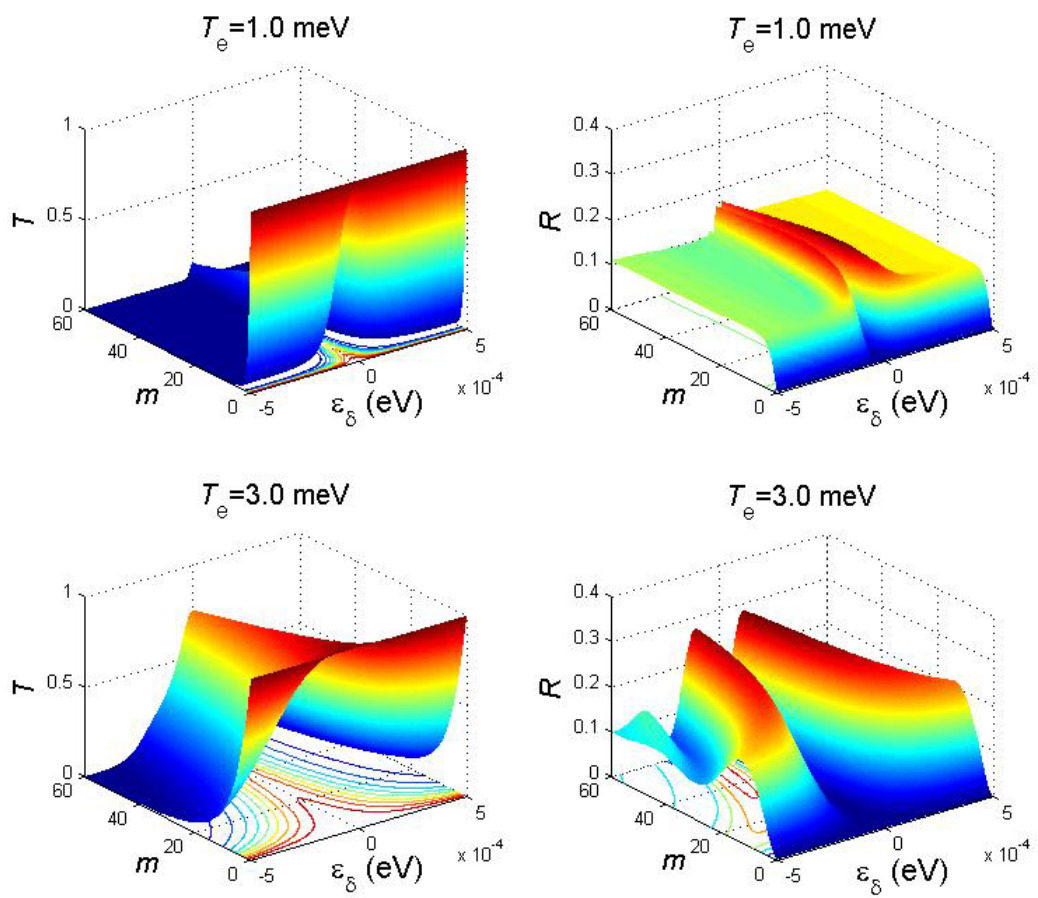

Figure 11. The tunable dispersion characteristics of the reflection and transmission spectrum of the thin film, of which the thickness is defined as $d=2 m \lambda_{0} /\left(4 \sqrt{\varepsilon_{b}}\right)$. When the incident probe frequency detuning $\varepsilon_{\delta}=\hbar \delta=\hbar \omega_{p}-\hbar \omega_{10}=0$ and the gate voltage $T_{e}=3.0 \mathrm{meV}$, the EIT effect can occur, and hence the transmittance $T$ increases and the reflectance $R$ decreases.
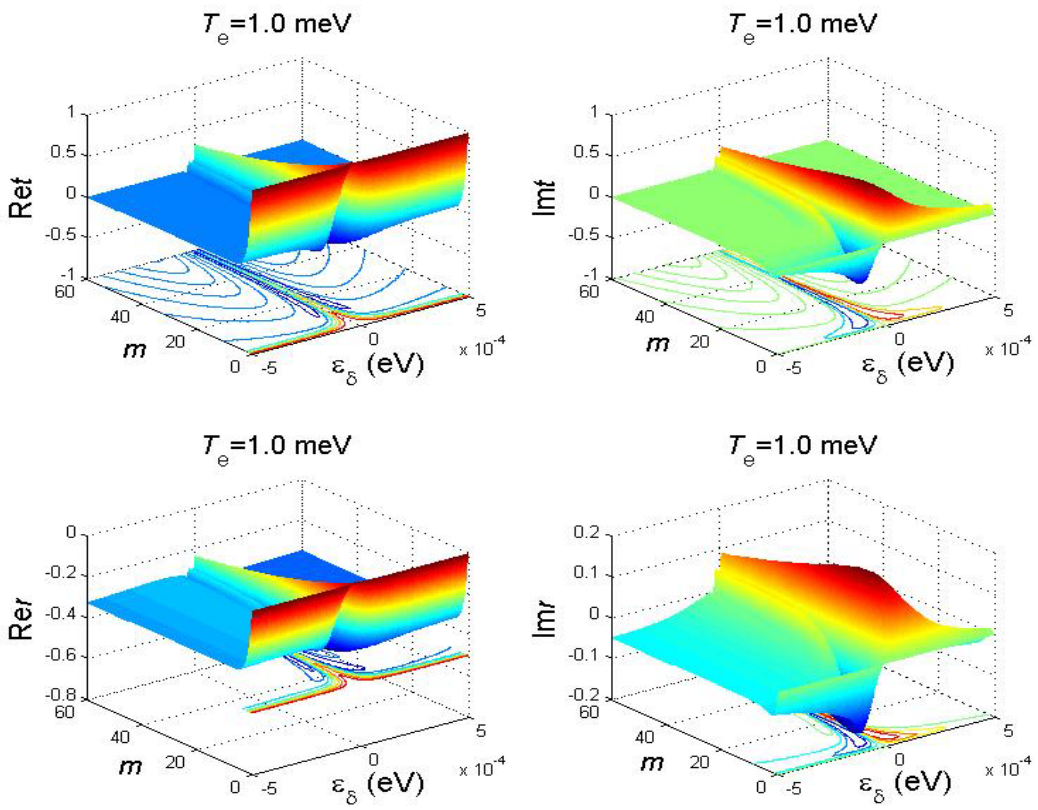

Figure 12. The tunable dispersion characteristics of the reflection and transmission spectrum of the thin film, of which the thickness is defined as $d=2 m \lambda_{0} /\left(4 \sqrt{\varepsilon_{b}}\right)$ when the gate voltage is $T_{e}=1.0 \mathrm{meV}$. Since the gate voltage is relatively small, the resonant tunneling effect of the probe light cannot be exhibited. 

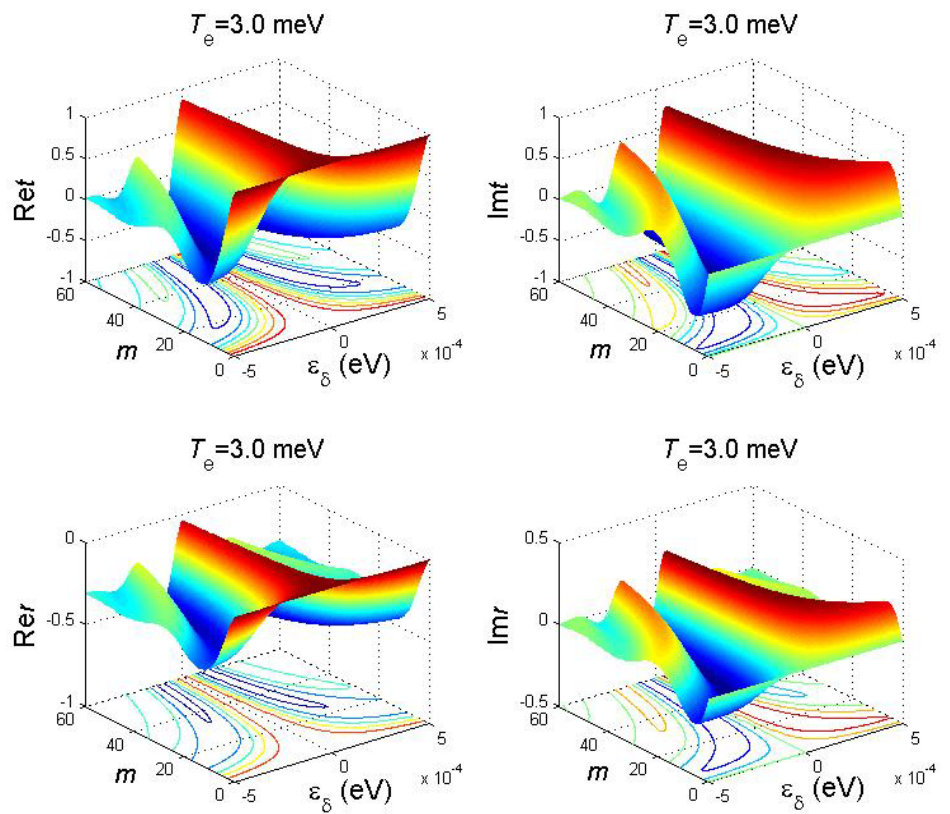

Figure 13. The tunable dispersion characteristics of the reflection and transmission spectrum of the thin film, of which the thickness is defined as $d=2 m \lambda_{0} /\left(4 \sqrt{\varepsilon_{b}}\right)$ when the gate voltage is $T_{e}=3.0 \mathrm{meV}$. Since the gate voltage becomes large, the resonant tunneling effect of the probe light can be exhibited at the probe frequency detuning $\varepsilon_{\delta}=\hbar \delta=\hbar \omega_{p}-\hbar \omega_{10}=0$.
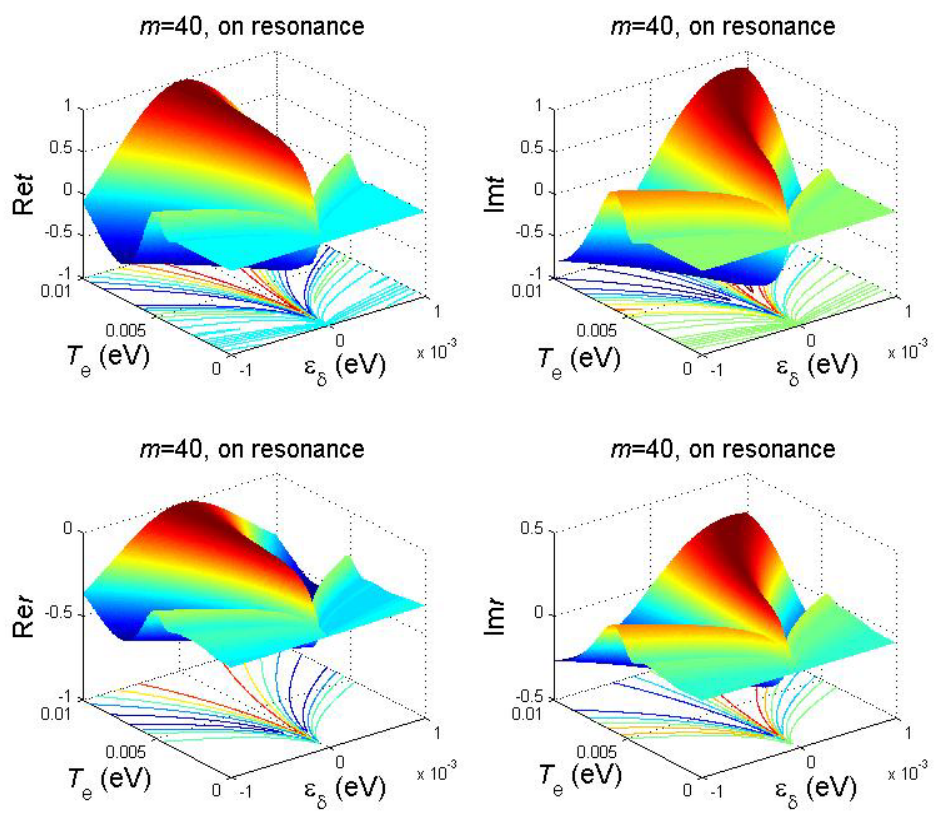

Figure 14. The tunable dispersion characteristics of the reflection and transmission spectrum of the thin film, of which the thickness is defined as $d=2 m \lambda_{0} /\left(4 \sqrt{\varepsilon_{b}}\right)$ with $m=40$. In the case of on resonance, when the gate voltage $T_{e}$ is large at the probe frequency detuning $\varepsilon_{\delta}=\hbar \delta=\hbar \omega_{p}-\hbar \omega_{10}=0$, the EIT of the probe field can be exhibited, and hence the resonant tunneling of the probe field will occur, i.e., the reflectance $R$ is small and the transmittance $T$ approaches unity. 

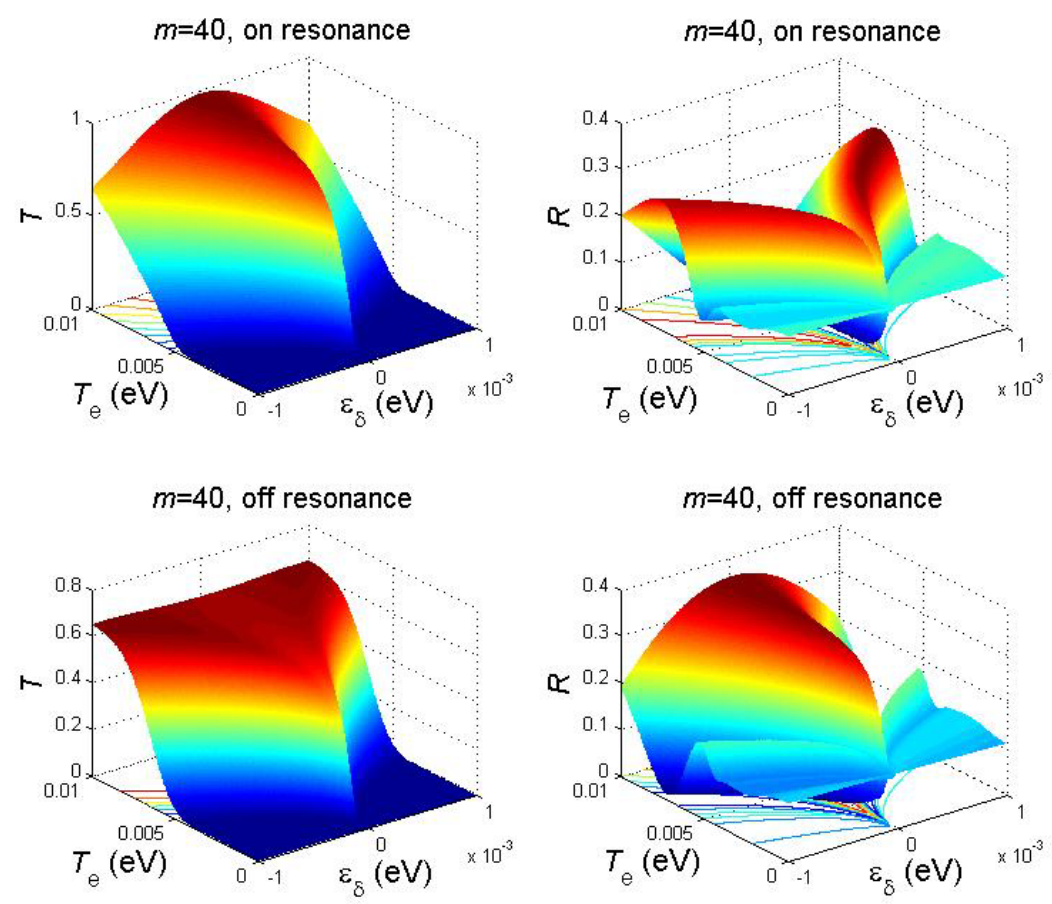

Figure 15. The tunable dispersion characteristics of the reflection and transmission spectrum of the thin film, of which the thickness is defined as $d=2 m \lambda_{0} /\left(4 \sqrt{\varepsilon_{b}}\right)$ (on resonance) and $d=(2 m+1) \lambda_{0} /\left(4 \sqrt{\varepsilon_{b}}\right)$ (off resonance) with $m=40$. In the case of on resonance, when the gate voltage $T_{e}$ is large at the probe frequency detuning $\varepsilon_{\delta}=\hbar \delta=\hbar \omega_{p}-\hbar \omega_{10}=0$, the EIT of the probe field can be exhibited, and hence the resonant tunneling of the probe field will occur, i.e., the reflectance $R$ is small and the transmittance $T$ approaches unity. However, in the case of off resonance, the reflectance $R$ increases and the transmittance $T$ decreases compared with the case of on resonance.

reflectance $R$ of the probe light increases and the transmittance $T$ decreases. This, therefore, means that the transmission and reflection spectrum of an incident electromagnetic field (probe field) can be tuned via the gate voltage that is applied on the quantum-dot molecular thin film.

\section{Transient Evolution of Controllable Tunneling of Light}

In the preceding sections we have addressed the steady optical properties of the quantum-dot molecular dielectric film, where the gate voltage has been turned on and hence all the physical quantities of the three-level quantum-dot molecular system are time-independent (i.e., steady). However, when the magnitude of gate voltage changes from one value to another one, e.g., the gate voltage is turned off before time $t<0$ and is turned on at $t=0$, this will lead to the transient evolutional optical behavior of the quantum-dot molecular dielectric film (i.e., the population in the present three-level quantum-dot molecular system will evolve from one steady state to another one). In this process, the transmission and reflection spectra of the probe light will also change from one 
steady case to the other one. Now we shall briefly discuss the transient solution of Equation (1). We will consider the approximate solution, of which the dynamical equations can be given by

$$
\begin{aligned}
& \mathrm{d} \dot{\rho}_{10} / \mathrm{d} t=-\left(\Gamma_{10}+i \Delta_{p}\right) \rho_{10}+\frac{i}{2}\left(\Omega_{p} \rho_{00}+\Omega_{c} \rho_{20}\right), \\
& \mathrm{d} \dot{\rho}_{20} / \mathrm{d} t=-\left[\Gamma_{20}+i\left(\Delta_{p}-\Delta_{c}\right)\right] \rho_{20}+\frac{i}{2} \Omega_{c}^{*} \rho_{10},
\end{aligned}
$$

where the small terms such as $-\frac{i}{2} \Omega_{p} \rho_{11}$ and $-\frac{i}{2} \Omega_{p} \rho_{21}$ have been ignored. We assume (ground state population) $\rho_{00} \approx 1$. These two equations in $(7)$ can be rewritten as a matrix equation

$$
\frac{\mathrm{d}}{\mathrm{d} t}\left(\begin{array}{l}
\dot{\rho}_{10} \\
\dot{\rho}_{20}
\end{array}\right)=\left(\begin{array}{cc}
-\left(\Gamma_{10}+i \Delta_{p}\right) & \frac{i}{2} \Omega_{c} \\
\frac{i}{2} \Omega_{c}^{*} & -\left[\Gamma_{20}+i\left(\Delta_{p}-\Delta_{c}\right)\right]
\end{array}\right)\left(\begin{array}{l}
\dot{\rho}_{10} \\
\dot{\rho}_{20}
\end{array}\right)+\left(\begin{array}{c}
\frac{i}{2} \Omega_{p} \\
0
\end{array}\right) .
$$

The steady solution when time $t \rightarrow+\infty \quad\left(d \dot{\rho}_{10} / d t=0, d \dot{\rho}_{20} / d t=0\right)$ is given by

$$
\begin{aligned}
& \dot{\rho}_{10}^{(f)}=\frac{\frac{1}{2}\left[-\left(\Delta_{p}-\Delta_{c}\right)+i \Gamma_{20}\right] \Omega_{p}}{\left(\Gamma_{10}+i \Delta_{p}\right)\left[\Gamma_{20}+i\left(\Delta_{p}-\Delta_{c}\right)\right]+\frac{1}{4} \Omega_{c}^{*} \Omega_{c}}, \\
& \dot{\rho}_{20}^{(f)}=\frac{\frac{i}{2} \Omega_{c}^{*} \dot{\rho}_{10}^{(f)}}{\Gamma_{20}+i\left(\Delta_{p}-\Delta_{c}\right)} .
\end{aligned}
$$

We assume the time-dependent solution is of the form $\dot{\rho}_{10}(t)=c_{1} e^{\lambda t}$, $\dot{\rho}_{20}(t)=c_{2} e^{\lambda t}$. Then by substituting these solutions into Equation (8), we can obtain a matrix eigenvalue equation

$$
\left(\begin{array}{cc}
-\left(\Gamma_{10}+i \Delta_{p}\right) & \frac{i}{2} \Omega_{c} \\
\frac{i}{2} \Omega_{c}^{*} & -\left[\Gamma_{20}+i\left(\Delta_{p}-\Delta_{c}\right)\right]
\end{array}\right)\left(\begin{array}{l}
c_{1} \\
c_{2}
\end{array}\right)=\lambda\left(\begin{array}{l}
c_{1} \\
c_{2}
\end{array}\right)
$$

The determinant of the matrix is

$$
\operatorname{det}\left(\begin{array}{cc}
-\left(\Gamma_{10}+i \Delta_{p}\right)-\lambda & \frac{i}{2} \Omega_{c} \\
\frac{i}{2} \Omega_{c}^{*} & -\left[\Gamma_{20}+i\left(\Delta_{p}-\Delta_{c}\right)\right]-\lambda
\end{array}\right)=0 \text {. }
$$

This leads to the relation $\left[\Gamma_{20}+i\left(\Delta_{p}-\Delta_{c}\right)+\lambda\right]\left(\Gamma_{10}+i \Delta_{p}+\lambda\right)+\frac{1}{4} \Omega_{c}^{*} \Omega_{c}=0$, which can also be rewritten as $\lambda^{2}+b \lambda+c=0$. Here, the two coefficients are defined as

$$
\begin{aligned}
& b=\Gamma_{10}+\Gamma_{20}+i\left(2 \Delta_{p}-\Delta_{c}\right), \\
& c=\left[\Gamma_{20}+i\left(\Delta_{p}-\Delta_{c}\right)\right]\left(\Gamma_{10}+i \Delta_{p}\right)+\frac{1}{4} \Omega_{c}^{*} \Omega_{c} .
\end{aligned}
$$


The solution of $\lambda^{2}+b \lambda+c=0$ is $\lambda_{ \pm}=\frac{-b \pm \sqrt{b^{2}-4 c}}{2}$. Now from Equation (10), we can obtain the coefficients of the solutions $c_{1 \pm}=\frac{\frac{i}{2} \Omega_{c} c_{2 \pm}}{\Gamma_{10}+i \Delta_{p}+\lambda_{ \pm}}$. Then the general solution of the matrix Equation (8) appears to be in the form

$$
\left(\begin{array}{c}
\dot{\rho}_{10}(t) \\
\dot{\rho}_{20}(t)
\end{array}\right)=\left(\begin{array}{c}
\frac{i}{2} \Omega_{c} \\
\Gamma_{10}+i \Delta_{p}+\lambda_{+} \\
1
\end{array}\right) c_{2+} e^{\lambda_{+} t}+\left(\begin{array}{c}
\frac{i}{2} \Omega_{c} \\
\Gamma_{10}+i \Delta_{p}+\lambda_{-} \\
1
\end{array}\right) c_{2-} e^{\lambda_{-} t}+\left(\begin{array}{c}
\dot{\rho}_{10}^{(f)} \\
\dot{\rho}_{20}^{(f)}
\end{array}\right)
$$

Some coefficients $c_{2 \pm}$ can be determined by using the initial conditions (steady solution) at time $t<0$ when the gate voltage has been turned off. It can be found that the two eigenvalues $\lambda_{ \pm}$are complex numbers with negative real parts, the solution $\dot{\rho}_{10}(t)=c_{1} e^{\lambda t}+\dot{\rho}_{10}^{(f)}, \quad \dot{\rho}_{20}(t)=c_{2} e^{\lambda t}+\dot{\rho}_{20}^{(f)} \quad$ will oscillatorily decay to a new steady one, i.e., $\dot{\rho}_{10}^{(f)}$ and $\dot{\rho}_{20}^{(f)}$. The transient evolutional dynamics (i.e., turn-on dynamics) is essentially important for device design (e.g., photonic switching and transistors).

\section{Concluding Remarks}

Quantum coherence, which occurs in alkali-metal atomic vapor and semiconductor quantum-dot media, has been studied in the literature [1]-[12]. In this report, we have considered the quantum-dot molecule dielectric film to realize the quantum coherence following the work [18] of controlling the propagation of light via external gate voltage in dielectric film doped with quantum-dot molecules. The gate voltage driving the inner-dot tunneling can make the quantumdot molecule system exhibit quantum coherence-EIT. We study both on-and off-resonance tunneling behavior in the quantum-dot molecule dielectric film related to the EIT effect. Reflection and transmission spectra of an incident electromagnetic field (probe field) can be tuned by the gate voltage applied on the quantum-dot molecule system. Under the large gate voltage and on-resonance tunneling condition, the probe light will propagate without loss through the quantum-dot molecule dielectric film if the probe frequency detuning is zero. The effects associated with the interaction of light with quantum dots as well as quantum-dot coupling effect would find potential applications in photonic device design such as optical switching and photonic logic gates.

\section{Acknowledgements}

This work is supported in part by the Natural Science Foundations of Zhejiang Province (China) under Project No. LY16A040002, the National Natural Science Foundations of China under Grants Nos. 61605170 and 11174250. The authors are also grateful to the Foundations of "Program of Zhejiang Leading Team of Science and Technology Innovation”. 


\section{References}

[1] Harris, S.E. (1997) Electromagnetically Induced Transparency. Physics Today, 50, 36-42. https://doi.org/10.1063/1.881806

[2] Gandman, A., Chuntonov, L., Rybak, L. and Amitay, Z. (2007) Pulse-Bandwidth Dependence of Coherent Phase Control of Resonance-Mediated (2+1) Three-Photon Absorption. Physical Review A, 75, 031401(R).

https://doi.org/10.1103/PhysRevA.75.031401

[3] Zheltikov, A.M. (2006) Phase Coherence Control and Subcycle Transient Detection in Nonlinear Raman Scattering with Ultrashort Laser Pulses. Physical Review A, 74, 053403. https://doi.org/10.1103/PhysRevA.74.053403

[4] Shen, J.Q. (2008) Classical \& Quantum Optical Properties of Artificial Electromagnetic Media. Transworld Research Network, Kerala.

[5] Yao, J.Q., Wu, H.B. and Wang, H. (2003) The Transient Optical Properties in Four-Level Atomic Medium Induced by Quantum Interference Effect. Acta Sinica Quantum Optics, 9, 121-125.

[6] Cohen, J.L. and Berman, P.R. (1997) Amplification without Inversion: Understanding Probability Amplitudes, Quantum Interference, and Feynman Rules in a Strongly Driven System. Physical Review A, 55, 3900-3917. https://doi.org/10.1103/PhysRevA.55.3900

[7] Zhu, S.Y. and Scully, M.O. (1996) Spectral Line Elimination and Spontaneous Emission Cancellation via Quantum Interference. Physical Review Letters, 76, 388391. https://doi.org/10.1103/PhysRevLett.76.388

[8] Champenois, C., Morigi, G. and Eschner, J. (2006) Quantum Coherence and Population Trapping in Three-Photon Processes. Physical Review A, 74, 053404. https://doi.org/10.1103/PhysRevA.74.053404

[9] Krowne, C.M. and Shen, J.Q. (2009) Dressed-State Mixed-Parity Transitions for Realizing Negative Refractive Index. Physical Review A, 79, 023818(11).

[10] Abdumalikov Jr., A.A., Astafiev, O., Zagoskin, A.M., Pashkin, Yu.A., Nakamura, Y. and Tsai, J.S. (2010) Electromagnetically Induced Transparency on a Single Artificial Atom. Physical Review Letters, 104, 193601. https://doi.org/10.1103/PhysRevLett.104.193601

[11] Palui, G., Aldeek, F., Wang, W. and Mattoussi, H. (2015) Strategies for Interfacing Inorganic Nanocrystals with Biological Systems Based on Polymer-Coating. Chemical Society Reviews, 44, 193-227. https://doi.org/10.1039/C4CS00124A

[12] Kruchinin, S.Yu., Rukhlenko, I.D., Baimuratov, A.S., Leonov, M.Yu., Turkov, V.K., Gunko, Y.K., Baranov, A.V. and Fedorov, A.V. (2014) Photoluminescence of a Quantum-Dot Molecule. Journal of Applied Physics, 117, 014306. https://doi.org/10.1063/1.4905258

[13] Unold, T., Mueller, K., Lienau, C., Elsaesser, T. and Wieck, A.D. (2005) Optical Control of Excitons in a Pair of Quantum Dots Coupled by the Dipole-Dipole Interaction. Physical Review Letters, 94, 137404. https://doi.org/10.1103/PhysRevLett.94.137404

[14] Stinaff, E.A., Scheibner, M., Bracker, A.S., Ponomarev, I.V., Korenev, V.L., Ware, M.E., Doty, M.F., Reinecke, T.L. and Gammon, D. (2006) Optical Signatures of Coupled Quantum Dots. Science, 311, 636-639. https://doi.org/10.1126/science.1121189

[15] Robledo, L., Elzerman, J., Jundt, G., Atature, M., Hogele, A., Falt, S. and Imamoglu, 
A. (2008) Conditional Dynamics of Interacting Quantum Dots. Science, 320, 772775. https://doi.org/10.1126/science.1155374

[16] Silvi, S. and Credi, A. (2015) Luminescent Sensors Based on Quantum Dot-Molecule Conjugates. Chemical Society Reviews, 44, 4275-4289. https://doi.org/10.1039/C4CS00400K

[17] Villas-Boas, J.M., Govorov, A.O. and Ulloa, S.E. (2004) Coherent Control of Tunneling in a Quantum Dot Molecule. Physical Review B, 69, 125342. https://doi.org/10.1103/PhysRevB.69.125342

[18] Zohravi, L.E. and Mahmoudi, M. (2014) Voltage-Controlled Transmission through a Dielectric Slab Doped with Quantum Dot Molecules. Chinese Optics Letters, 12, 042601. https://doi.org/10.3788/COL201412.042601

[19] Hamedi, H.R. and Mehmannavaz, M.R. (2015) Behavior of Optical Bistability in Multifold Quantum Dot Molecules. Laser Physics, 25, 025403. https://doi.org/10.1088/1054-660X/25/2/025403

[20] Wang, Z., Zhen, S., Wu, X., Zhu, J., Cao, Z. and Yu, B. (2013) Controllable Optical Bistability via Tunneling Induced Transparency in Quantum Dot Molecules. Optics Communications, 304, 7-10. https://doi.org/10.1016/j.optcom.2013.04.024

[21] Borges, H.S., Sanz, L., Villas-Bôas, J.M., Diniz, N.O.O. and Alcalde, A.M. (2012) Tunneling Induced Transparency and Slow Light in Quantum Dot Molecules. Physical Review B, 85, 115425. https://doi.org/10.1103/PhysRevB.85.115425

[22] Hamedi, H.R. (2014) Ultra-Slow Propagation of Light Located in Ultra-Narrow Transparency Windows through Four Quantum Dot Molecules. Laser Physics Letters, 11, 085201. https://doi.org/10.1088/1612-2011/11/8/085201

[23] Tian, S.-C., Tong, C.-Z., Wang, C.-L. and Ning, Y.-Q. (2014) Effects of Spontaneously Generated Coherence on Resonance Fluorescence from Triple Quantum Dot Molecules. Journal of Luminescence, 153, 169-176.

https://doi.org/10.1016/j.jlumin.2014.03.034

[24] Daniels, J.M., Machnikowski, P. and Kuhn, T. (2013) Excitons in Quantum Dot Molecules: Coulomb Coupling, Spin-Orbit Effects and Phonon-Induced Line Broadening. Physical Review B, 88, 205307. https://doi.org/10.1103/PhysRevB.88.205307

[25] Borges, H.S., Sanz, L., Villas-Bôas, J.M. and Alcalde, A.M. (2013) Quantum Interference and Control of the Optical Response in Quantum Dot Molecules. Applied Physics Letters, 103, 222101. https://doi.org/10.1063/1.4833239

[26] Khoshnegar, M., Jafari-Salim, A., Ansari, M.H. and Majedi, A.H. (2014) Towards Tripartite Hybrid Entanglement in Quantum Dot Molecules. New Journal of Physics, 16, 023019. https://doi.org/10.1088/1367-2630/16/2/023019

[27] Shen, J.Q. and Zeng, R.X. (2017) Quantum-Coherence-Assisted Tunable On- and Off-Resonance Tunneling through a Quantum-Dot-Molecule Dielectric Film. Journal of the Physical Society of Japan, 86, 024401. https://doi.org/10.7566/JPSJ.86.024401

[28] Cheng, D.K. (1989) Field and Wave Electromagnetics. 2nd Edition, Addison-Wesley, New York. 
Submit or recommend next manuscript to SCIRP and we will provide best service for you:

Accepting pre-submission inquiries through Email, Facebook, LinkedIn, Twitter, etc. A wide selection of journals (inclusive of 9 subjects, more than 200 journals)

Providing 24-hour high-quality service

User-friendly online submission system

Fair and swift peer-review system

Efficient typesetting and proofreading procedure

Display of the result of downloads and visits, as well as the number of cited articles Maximum dissemination of your research work

Submit your manuscript at: http://papersubmission.scirp.org/

Or contactopj@scirp.org 